\title{
Carga, acceso y disparidades en la enfermedad renal
}

\author{
Burden, access, and disparities in kidney disease
}

\begin{abstract}
Deidra C. Crews ${ }^{1,2,3 *}$, Aminu K. Bello ${ }^{4}$ y Gamal Saadi5; World Kidney Day Steering Committee ${ }^{6 * *}$
${ }^{1}$ Division of Nephrology, Department of Medicine, Johns Hopkins University School of Medicine, Baltimore, Maryland, USA; ${ }^{2}$ Welch Center for Prevention, Epidemiology and Clinical Research, Johns Hopkins Medical Institutions, Baltimore, Maryland, USA; ${ }^{3}$ Johns Hopkins Center for Health Equity, Johns Hopkins Medical Institutions, Baltimore, Maryland, USA; ${ }^{4}$ Division of Nephrology \& Transplant Immunology, Department of Medicine, University of Alberta, Edmonton, Canada; ${ }^{5}$ Nephrology Unit, Department of Internal Medicine, Faculty of Medicine, Cairo University, Giza, Egypt
\end{abstract}

Este artículo se ha publicado en Kidney International y reimpreso concurrentemente en diversas revistas. Los artículos cubren conceptos y redacción idénticos, pero pueden tener variaciones mínimas en el estilo, ortografía, detalles y longitud del manuscrito para mantener el estilo de cada revista. Cualquiera de estas versiones puede ser usada para citar este artículo.

**Todos los autores contribuyeron de igual manera en la concepción, preparación y edición del manuscrito.

\#Vea el Apéndice para la lista de los miembros del Comité Directivo del Día Mundial del Riñón.

○) World Kidney Day 2019 Steering Committee

La enfermedad renal es un problema global de salud pública que afecta a más de 750 millones de personas alrededor de todo el mundo'. La carga de la enfermedad renal varía sustancialmente a lo largo del planeta, al igual que su detección y tratamiento. A pesar de que la magnitud y el impacto de la enfermedad renal está mejor definida en los países desarrollados, evidencia reciente sugiere que los países en desarrollo tienen una carga de la enfermedad similar o incluso mayor que los primeros ${ }^{2}$.

En muchos escenarios, las tasas de enfermedad renal y la provisión de su cuidado están definidas por factores socioeconómicos, culturales y políticos, lo que ocasiona disparidades significativas en la carga de la enfermedad, incluso en países desarrollados ${ }^{3}$. Estas disparidades existen a lo largo de todo el espectro de la enfermedad renal-desde los esfuerzos preventivos para limitar el desarrollo de la lesión renal aguda (LRA) 0 de la enfermedad renal crónica (ERC), al tamizaje para enfermedad renal entre las personas con alto riesgo de desarrollarla, o al acceso a cuidado y tratamiento subespecializado de la falla renal con terapia de reemplazo renal (TRR). El Día Mundial del Riñón 2019 ofrece una oportunidad para hacer conciencia de la enfermedad renal y resaltar las disparidades en su carga y el estado actual de la capacidad global para su prevención y manejo. En esta editorial, resaltamos estas disparidades y hacemos énfasis en el rol de las políticas públicas y las estructuras organizacionales en su atención. También destacamos las oportunidades de mejorar nuestro entendimiento de las disparidades en la enfermedad renal, la mejor forma de que puedan ser reducidas, y como canalizar los esfuerzos enfocados a alcanzar una salud renal con equidad a lo largo del planeta.

\section{Carga de la enfermedad renal}

La disponibilidad de datos que reflejen la carga completa de la enfermedad renal varían sustancialmente debido a prácticas limitadas o inconsistentes en recolección de datos y vigilancia en todo el mundo (Tabla 1) 4 . Mientras que diversos países tienen sistemas nacionales de recolección de datos, particularmente

\footnotetext{
Correspondencia:

*Deidra C. Crews

E-mail: dcrews1@jhmi.edu

Fecha de recepción: 11-02-2019

Fecha de aceptación: 13-02-2019

DOI: 10.24875/NEFRO.M18000004

Disponible en internet: 09-08-2019

Nefro Latinoam. 2019;16:1-9 www.nefrologialatinoamericana.com

2444-9032/C World Kidney Day 2019 Steering Committee. Publicado por Permanyer México SA de CV. Este es un artículo Open Access bajo la licencia CC BY-NC-ND (http://creativecommons.org/licenses/by-nc-nd/4.0/).
} 
Nefro Latinoam. 2019;16

Tabla 1. Brechas de la enfermedad renal crónica por grupos de países del Banco Mundial

\begin{tabular}{|c|c|c|c|c|}
\hline Atención ERC & $\begin{array}{c}\text { Países de } \\
\text { ingresos bajos (\%) }\end{array}$ & $\begin{array}{l}\text { Países de ingresos } \\
\text { medios-bajos (\%) }\end{array}$ & $\begin{array}{l}\text { Países de ingresos } \\
\text { medios-altos (\%) }\end{array}$ & $\begin{array}{c}\text { Países de } \\
\text { ingresos altos (\%) }\end{array}$ \\
\hline $\begin{array}{l}\text { Reconocimiento gubernamental a la ERC como } \\
\text { una prioridad de salud }\end{array}$ & 59 & 50 & 17 & 29 \\
\hline $\begin{array}{l}\text { El gobierno provee financiamiento a todos los } \\
\text { aspectos del cuidado de la ERC }\end{array}$ & 13 & 21 & 40 & 53 \\
\hline $\begin{array}{l}\text { Disponibilidad de guías de manejo y referencia } \\
\text { de la (internacional, nacional o regional) }\end{array}$ & 46 & 73 & 83 & 97 \\
\hline $\begin{array}{l}\text { Existencia actual de programas de detección } \\
\text { de la ERC }\end{array}$ & 6 & 24 & 24 & 32 \\
\hline Disponibilidad de registros de diálisis & 24 & 48 & 72 & 89 \\
\hline $\begin{array}{l}\text { Disponibilidad de centros académicos para el } \\
\text { manejo de ensayos clínicos renales }\end{array}$ & 12 & 34 & 62 & 63 \\
\hline
\end{tabular}

para enfermedad renal crónica terminal (ERCT) (p. ej., el United States Renal Data System, el Registro Latinoamericano de Diálisis y Trasplante Renal y el Australia and New Zealand Dialysis and Transplant Registry), los datos de alta calidad relacionados con ERC no en diálisis son limitados, y usualmente la calidad de los datos de ERCT varía mucho en diversos escenarios. Esta situación es de particular preocupación en países con ingresos bajos. Por ejemplo, un metaanálisis de 90 estudios en carga de ERC llevado a cabo en África mostró muy pocos estudios (únicamente el $3 \%$ ) con datos robustos ${ }^{5}$. La provisión de recursos y fuerza de trabajo adecuados para establecer y mantener sistemas de vigilancia (p. ej., programas de tamizaje y registros) es esencial y require una inversión sustancial ${ }^{6}$. El incorporar parámetros de vigilancia de la enfermedad renal en programas de prevención de enfermedades crónicas existentes podría incrementar los esfuerzos globales orientados a obtener información de alta calidad en la carga de la enfermedad renal y atender sus consecuencias.

Adicional a la necesidad de sistemas de vigilancia funcionales, la importancia global de la enfermedad renal (incluyendo LRA y ERC) aún tiene que ser reconocida ampliamente: es una enfermedad descuidada en la agenda global de políticas de salud. Por ejemplo, el Plan de Acción Global para la Prevención y Control de Enfermedades No Comunicables (ENC) de la Organización Mundial de la Salud (OMS) (2013) se enfoca en la enfermedad cardiovascular, cáncer, enfermedades respiratorias crónicas y diabetes, pero no en la enfermedad renal, a pesar de los esfuerzos hechos por diversos organismos importantes como la Sociedad Internacional de Nefrología y la Federación Internacional de Fundaciones Renales a través de actividades como el Día Mundial del Riñón. Esta situación es preocupante debido a que, según estimaciones del Estudio Global de la Carga de la Enfermedad, en 2015, 1.2 millones de personas murieron a causa de la $\mathrm{ERC}^{7}, \mathrm{y}$ más de 2 millones de personas murieron en 2010 debido a que no tuvieron acceso a diálisis. Se estima que otros 1.7 millones de personas mueren anualmente por LRA $^{8,9}$. Es posible, por lo tanto, que la enfermedad renal pueda contribuir a más muertes que las cuatro principales ENC objetivo del actual Plan de Acción para ENC.

\section{Factores de riesgo para enfermedad renal}

Los datos obtenidos en décadas recientes han ligado factores genéticos, ambientales, sociodemográficos y clínicos a la enfermedad renal. Se sabe que la carga poblacional de enfermedad renal se correlaciona con factores definidos socialmente en la mayoría de sociedades alrededor del mundo. Este fenómeno está mejor documentado en países con ingresos altos, donde los grupos con minoría racial o étnica y las personas de bajo nivel socioeconómico llevan una alta carga de la enfermedad. Datos extensos han demostrado que las minorías raciales y étnicas (p. ej., afroamericanos en los Estados Unidos, los grupos aborígenes en Canadá y Australia, los indo-asiáticos en el Reino Unido, y otros) se ven 
afectados desproporcionadamente por la enfermedad renal avanzada y progresiva ${ }^{10-12}$. La asociación del estado socioeconómico y el riesgo de ERC progresiva y eventualmente falla renal también se ha descrito extensamente, y son las personas de bajo estatus socioeconómico quienes llevan la mayor carga ${ }^{13,14}$.

Trabajos recientes han asociado a variantes de riesgo de apolipoproteína $L 1^{15,16}$ con un incremento de la carga de enfermedad renal entre personas con ancestros africanos. En América Central y el sureste de México, la nefropatía mesoamericana (también referida como ERC de etiología desconocida) ha emergido como una causa importante de enfermedad renal. Si bien se han estudiado exposiciones múltiples por su papel potencial en la ERC de etiología desconocida, la deshidratación recurrente y el estrés por calor son denominadores comunes en la mayoría de los casos $^{17}$. También se han identificado otros factores de riesgo para la enfermedad renal y la progresión de la ERC que pueden afectar de manera desproporcionada a los grupos socialmente desfavorecidos, que incluyen tasas dispares y un control deficiente de los factores de riesgo clínicos como la diabetes y la hipertensión, así como los comportamientos de estilo de vida.

La diabetes es la principal causa de enfermedad renal avanzada en todo el mundo ${ }^{18}$. En 2016, 1 de cada 11 adultos en todo el mundo padecía diabetes y más del $80 \%$ vivía en países con ingresos bajos y medios ${ }^{19}$ donde los recursos para una atención óptima son limitados. También se estima que la hipertensión afecta a 1,000 millones de personas en todo el mundo ${ }^{20}$ y es la segunda causa atribuible de $\mathrm{ERC}^{18}$. El control de la hipertensión es importante para disminuir la progresión de la ERC y disminuir el riesgo de mortalidad entre las personas con o sin ERC. La hipertensión está presente en más del $90 \%$ de las personas con enfermedad renal avanzada $^{18}$, sin embargo, las minorías raciales/étnicas y las personas con ingresos bajos con ERC que viven en países con ingresos altos tienen un control de la presión arterial más deficiente que quienes tienen mayores ventajas sociales ${ }^{21}$.

Los hábitos de estilo de vida, incluidos los patrones dietéticos, están muy influenciados por el estatus socioeconómico. En los últimos años, varios patrones dietéticos saludables se han asociado con resultados de ERC favorables ${ }^{22}$. Las personas con ingresos bajos a menudo tienen dificultades para seguir una dieta saludable, lo que puede aumentar su riesgo de enfermedad renal ${ }^{23-25}$. Las personas de bajo nivel socioeconómico a menudo experimentan «inseguridad alimentaria» (p. ej., acceso limitado a alimentos nutritivos asequibles), que es un factor de riesgo para la ERC ${ }^{26}$ y la progresión a insuficiencia renal ${ }^{27}$. En los países con ingresos bajos, la inseguridad alimentaria puede llevar a la desnutrición e inanición, lo que tiene implicaciones para el individuo y, en el caso de las mujeres en edad fértil, puede llevar a sus hijos a tener un bajo peso al nacer y secuelas relacionadas, incluida la $\mathrm{ERC}^{28}$. Las tasas de desnutrición alcanzan el 35\% o más en países como Haití, Namibia y Zambia ${ }^{29}$. Sin embargo, en los países con ingresos altos, la inseguridad alimentaria se ha asociado con la sobrealimentación, y las personas con inseguridad alimentaria tienen un mayor riesgo de sobrepeso y obesidad ${ }^{30,31}$. Además, la inseguridad alimentaria se ha asociado con varias enfermedades relacionadas con la dieta, incluyendo diabetes e hipertensión.

\section{Lesión renal aguda}

La LRA es una condición muy poco detectada que se estima que ocurre en el $8 \%$ a $16 \%$ de los ingresos hospitalarios ${ }^{32}$ y ahora está bien establecida como un factor de riesgo para la $\mathrm{ERC}^{33}$.Las disparidades en el riesgo de padecer LRA también son comunes, siguiendo un patrón similar al observado en personas con $\mathrm{ERC}^{34}$. La LRA relacionada con nefrotoxinas, medicamentos alternativos (tradicionales), agentes infecciosos y hospitalizaciones y procedimientos relacionados es más pronunciada en los países con ingresos bajos y medianos, lo que contribuye a un mayor riesgo de mortalidad y ERC en esos entornos ${ }^{35}$. Es importante destacar que la mayoría de los casos anuales de LRA en todo el mundo ( $85 \%$ de más de 13 millones de casos) se presentan en países con ingresos bajos y medios-bajos, lo que lleva a 1,4 millones de muertes ${ }^{36}$.

\section{Políticas de salud y financiamiento del cuidado de la enfermedad renal}

Debido a la naturaleza compleja y costosa de la atención de la enfermedad renal, su provisión está estrechamente relacionada con las políticas públicas y la situación financiera de cada país. Por ejemplo, el producto interno bruto se correlaciona con una menor proporción de diálisis-a-trasplante, lo que sugiere mayores tasas de trasplante de riñón en las naciones financieramente más solventes. En varios países con ingresos altos, la atención médica universal es proporcionada por el gobierno e incluye la ERC y la ERCT. En otros países, como EE.UU., la atención de ERCT se financía con fondos públicos para los ciudadanos; 
sin embargo, el tratamiento óptimo de la ERC y sus factores de riesgo pueden no ser accesibles para las personas que carecen de seguro de salud, y la atención regular a los inmigrantes indocumentados con enfermedad renal no está cubierta ${ }^{37}$. En los países con ingresos bajos y medios-bajos, ni la atención de la ERC o de la ERCT pueden ser financiadas con fondos públicos, y los esfuerzos de prevención de la ERC a menudo son limitados. En varios de estos países han surgido colaboraciones entre los sectores público y privado para proporcionar fondos para la TRR. Por ejemplo, en Karachi, Pakistán, existe un programa de diálisis y trasplante de riñón a través de fondos conjuntos de la comunidad y el gobierno desde hace más de 25 años ${ }^{38}$.

En muchos entornos, las personas con ERC avanzada que no cuentan con fondos del sector público o privado, o que cuentan con una financiación limitada para estos servicios, asumen una carga financiera sustancial. Una revisión sistemática de 260 estudios que incluyeron pacientes de 30 países identificó desafíos significativos, incluida la atención fragmentada de duración indeterminada, dependencia de la atención de emergencia y el temor a eventos catastróficos de la vida debido a la capacidad financiera reducida para resistirlos ${ }^{39}$. Autores de otro estudio realizado en México encontraron que los pacientes y las familias estaban agobiados por tener que navegar por múltiples estructuras de atención social y de salud, negociar tratamientos y costos, financiar su atención médica y manejar la información de salud ${ }^{40}$. Los desafíos pueden ser aún mayores para las familias de niños con ERCT, porque muchas regiones carecen de centros de atención pediátrica cualificados.

\section{Organización y estructuras para la atención de la enfermedad renal}

La falta de reconocimiento y, por lo tanto, la ausencia de un plan de acción global para la enfermedad renal explican en parte la variación sustancial en las estructuras y la capacidad de atención renal en todo el mundo. Esta situación ha dado lugar a variaciones en las prioridades gubernamentales, los presupuestos de atención médica, las estructuras de atención y la disponibilidad de recursos humanos ${ }^{41}$. Se necesitan esfuerzos de promoción efectivos y sostenibles a nivel mundial, regional y nacional para que la enfermedad renal sea reconocida y se incluya en la agenda política global.
En 2017, la Sociedad Internacional de Nefrología recopiló datos sobre la capacidad a nivel de país para la prestación de atención renal mediante una encuesta, el Global Kidney Health Atlas ${ }^{4}$, que se alineó con los componentes básicos de la OMS de un sistema de salud. El Global Kidney Health Atlas destaca el conocimiento limitado de la enfermedad renal y sus consecuencias y las inequidades persistentes en los recursos necesarios para enfrentar la carga de la enfermedad renal en todo el mundo. Por ejemplo, la ERC fue reconocida como una prioridad de atención médica por el gobierno en solo el $36 \%$ de los países que participaron en esta encuesta. La prioridad estaba inversamente relacionada con el nivel de ingresos: la ERC era una prioridad de atención médica en más de la mitad de los países con ingresos bajos y medios-bajos, pero en menos del $30 \%$ de los países con ingresos medios-altos y altos.

Con respecto a la capacidad y los recursos para el cuidado renal, muchos países aún carecen de acceso a métodos de diagnóstico básico, como una fuerza de trabajo de nefrología capacitada, acceso universal a la atención primaria de salud y tecnologías de TRR. Los países con ingresos bajos y con ingresos medios-bajos, especialmente en África, tenían servicios limitados para el diagnóstico, manejo y monitoreo de la ERC en el nivel de atención primaria, solo el $12 \%$ tenía la determinación de creatinina sérica, incluida la tasa estimada de filtración glomerular. El 29\% de los países con ingresos bajos tuvo acceso a un análisis de orina cualitativo mediante el uso de tiras reactivas de orina; sin embargo, ningún país con ingresos bajos tuvo acceso a la medición de la relación de albúmina/creatinina en orina o de la tasa de proteína/creatinina en orina en el nivel de atención primaria. En todos los países del mundo, la disponibilidad de servicios en el nivel de atención secundaria/terciaria fue considerablemente mayor que en el nivel de atención primaria (Figs. 1A y B) ${ }^{4,42}$.

\section{Terapias de reemplazo renal}

La distribución de las tecnologías TRR varió ampliamente. En la superficie, todos los países informaron tener servicios de hemodiálisis de largo plazo, y más del $90 \%$ de los países informaron que tenían servicios de hemodiálisis de corto plazo. Sin embargo, el acceso y la distribución de la TRR entre países y regiones fue altamente inequitativo, lo que a menudo requería gastos prohibitivos, particularmente en regiones con ingresos bajos. Por ejemplo, más del $90 \%$ de los países con ingresos medios-altos y altos reportaron tener servicios 


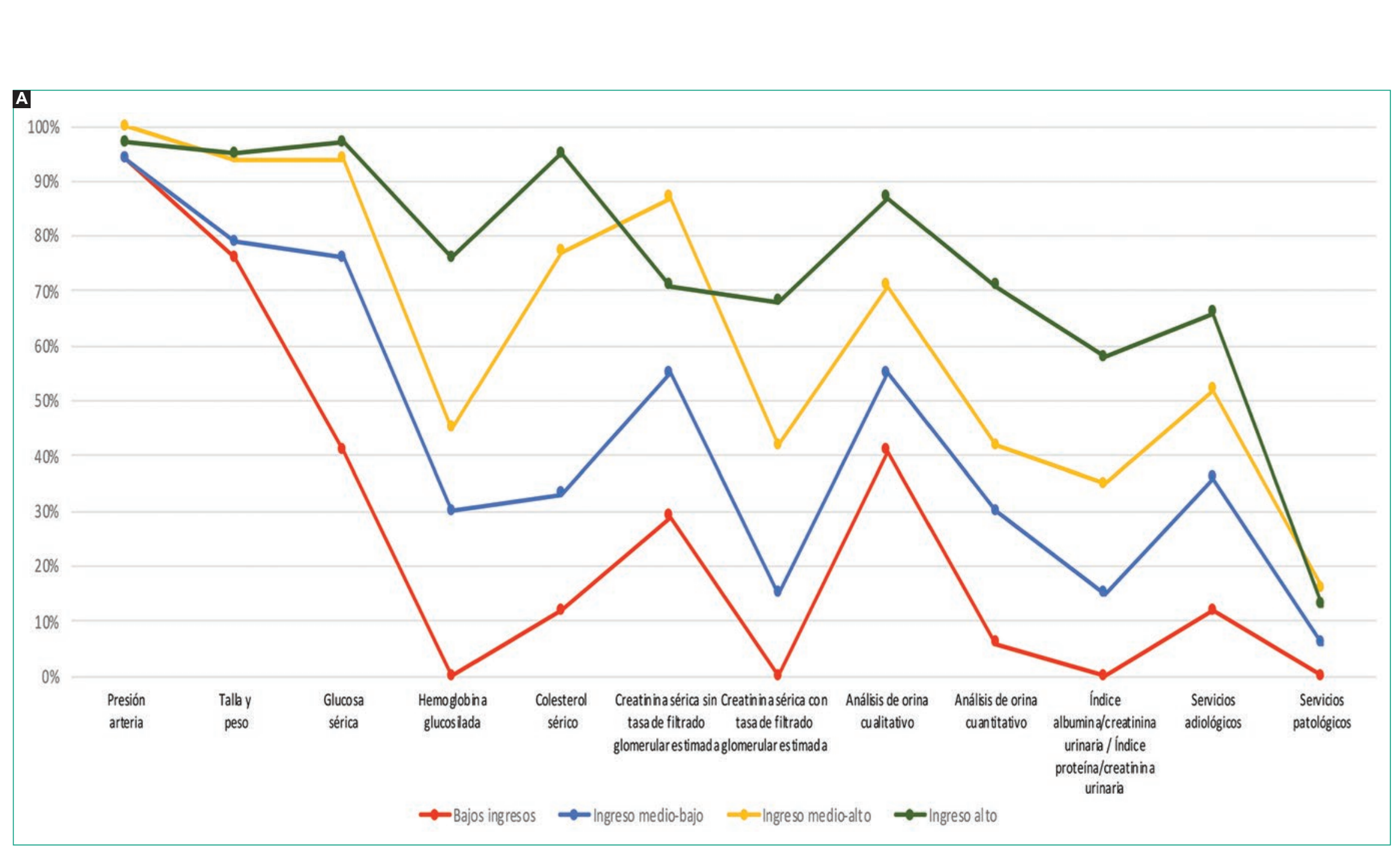

Figura 1A. Servicios de salud para la identificación y manejo de la enfermedad renal crónica por nivel de ingresos de cada país (datos A: primer nivel (p. ej., instalaciones básicas de salud a nivel comunitario [clínicas, dispensarios y hospitales locales pequeños]). 


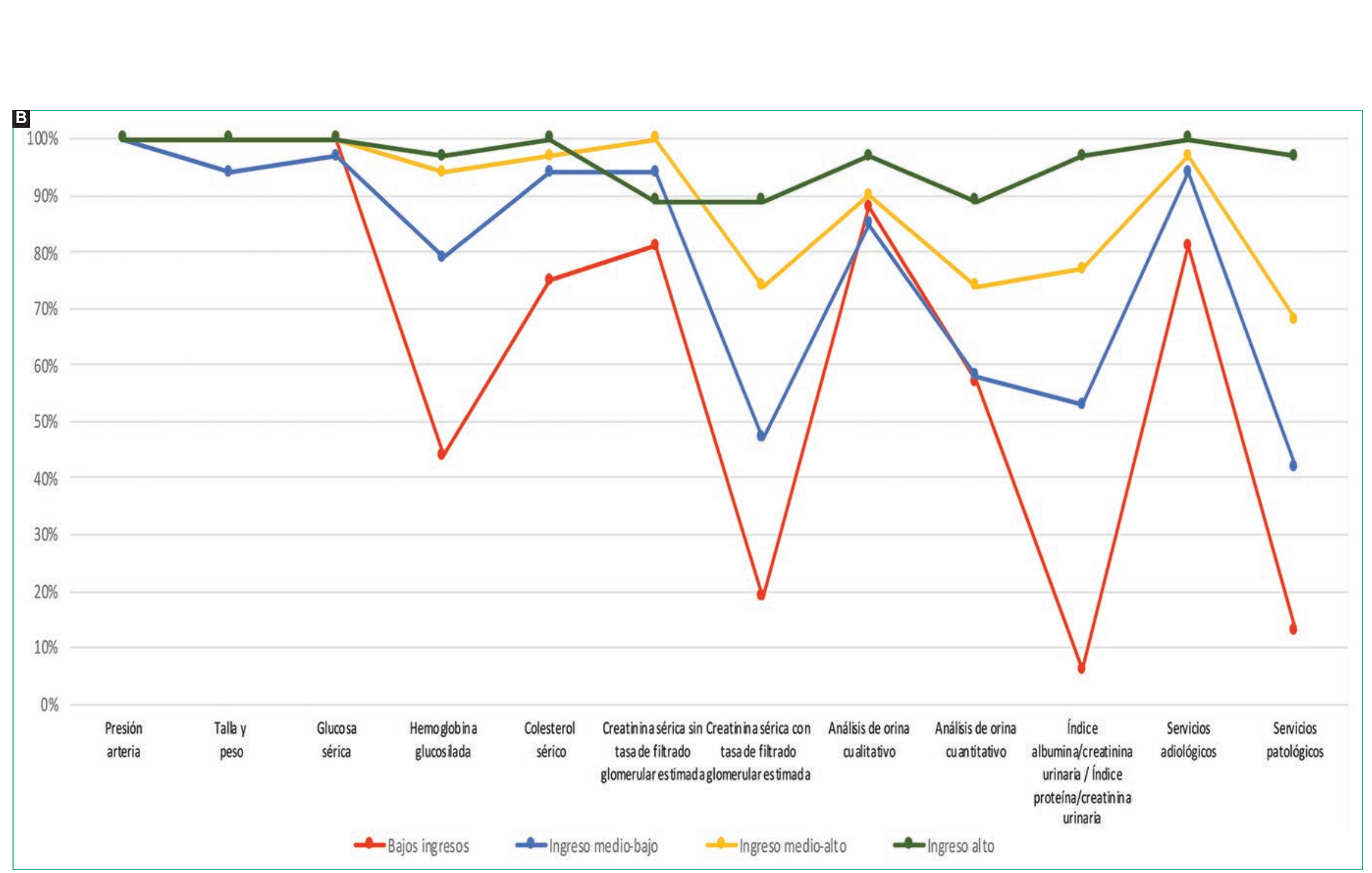

Figura 1B. Servicios de salud para la identificación y manejo de la enfermedad renal crónica por nivel de ingresos de cada país (datos de Bello, et al. ${ }^{4}$ y Htay, et al. ${ }^{42}$ ). B: segundo nivel y especialidad (p. ej., instalaciones de salud a un mayor nivel que el primario [clínicas, hospitales y centros académicosi). 


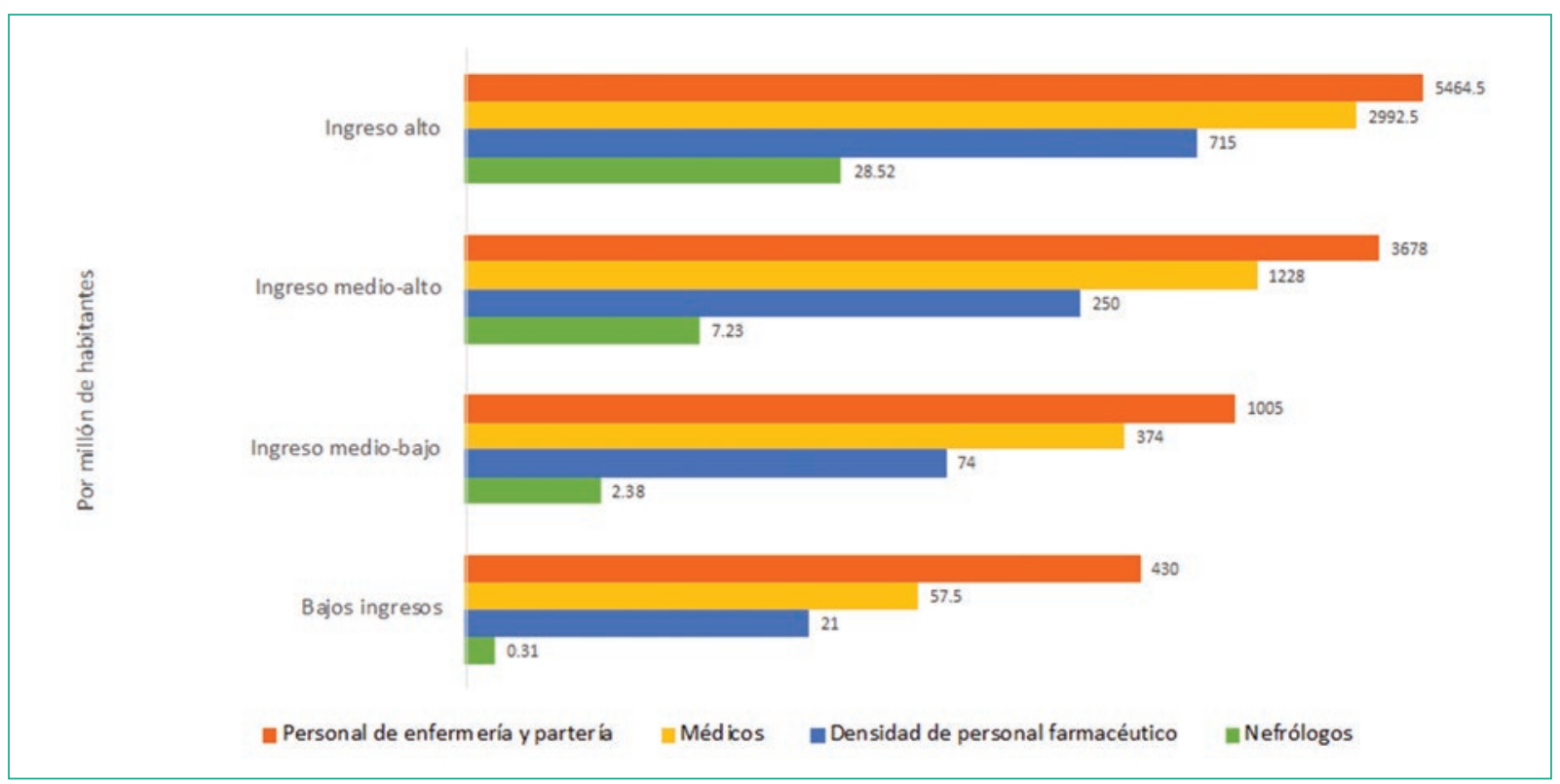

Figura 2. Disponibilidad de nefrólogos (densidad por millón de habitantes) comparada con médicos, enfermeras y personal de farmacia disponible por nivel de ingresos de cada país. El personal de farmacia incluye farmacéuticos, asistentes farmacéuticos, y técnicos farmacéuticos. Personal de enfermería y parteras incluye enfermeras y parteras profesionales, enfermeras y parteras auxiliares, enfermeras y parteras en entrenamiento y ocupaciones relacionadas, como enfermeras dentales. Se utilizó una escala logarítmica para el eje de las $x[\log (x+1)]$ debido al rango tan grande de la densidad de proveedores (datos de Bello, et al. ${ }^{4}$, Osman, et al. ${ }^{43}$ y la Organización Mundial de la Salud [para personal farmacéutico: http://apps.who.int/gho/data/view.main.PHARMS and http://apps.who.int/gho/data/node.mainamro.HWF?lang=en, para personal de enfermería y parteras: http://apps.who.int/gho/data/view.main.NURSES, para médicos: http://apps.who.int/gho/data/view.main.92000] $]^{44}$.

de diálisis peritoneal crónica, mientras que estos servicios estaban disponibles en el $64 \%$ y el $35 \%$ de los países con ingresos bajos y medios-bajos, respectivamente. En comparación, la diálisis peritoneal aguda tuvo la menor disponibilidad en todos los países. Más del $90 \%$ de los países con ingresos medios-altos y altos reportaron tener servicios de trasplante de riñón, y más del $85 \%$ de estos países informaron que tanto los donantes vivos como los donantes fallecidos son la fuente de los órganos. Como se esperaba, los países con ingresos bajos tenían la disponibilidad más baja de servicios de trasplante de riñón, solo el 12\% informaron disponibilidad, y los donantes vivos como la única fuente.

\section{Fuerza de trabajo para el cuidado renal}

También se observó una considerable variación internacional en la distribución de la fuerza de trabajo para la atención renal, en particular en los nefrólogos. La densidad más baja ( $<5$ nefrólogos por millón de habitantes) fue muy común en los países con ingresos bajos, mientras que la densidad más alta (>15 nefrólogos por millón de habitantes) se informó principalmente en los países con ingresos altos (Fig. 2) $)^{4,43,44}$. La mayoría de los países informaron que los nefrólogos son los principales responsables de la atención de la ERC y la LRA. Los médicos de atención primaria tenían más responsabilidad por la atención de la ERC que por la atención de la LRA, ya que el $64 \%$ de los países informaron que los médicos de atención primaria son los principales responsables de la atención de la ERC y el $35 \%$ informó que son responsables de la atención de la LRA. Los especialistas en cuidados intensivos fueron los principales responsables de la LRA en el $75 \%$ de los países, probablemente porque la LRA generalmente se trata en hospitales. Sin embargo, solo el $45 \%$ de los países con ingresos bajos informaron que los especialistas en cuidados intensivos eran los principales responsables de LRA, en comparación con el $90 \%$ de los países con ingresos altos; esta discrepancia puede deberse a una escasez general de especialistas en cuidados intensivos en países con ingresos bajos.

El número adecuado de nefrólogos en un país depende de muchos factores, entre los que se incluyen la 
necesidad, la prioridad y los recursos, y como tal no existe un estándar global con respecto a la densidad de los nefrólogos. En cualquier caso, la baja densidad demostrada en los países con ingresos bajos llama a la preocupación, ya que los nefrólogos son esenciales para proporcionar liderazgo en la atención de la enfermedad renal, y la falta de nefrólogos puede tener consecuencias adversas para las políticas y la práctica. Sin embargo, es bastante alentador que el número de nefrólogos y nefropatólogos esté aumentando en los países con ingresos bajos y medios-bajos, en parte gracias a los programas de becas apoyados por organizaciones internacionales de nefrología ${ }^{45}$. Es importante señalar que el papel del nefrólogo puede diferir según la estructura del sistema de atención médica. La estadística de densidad simplemente representa el número de nefrólogos por millón de habitantes y no proporciona ninguna indicación de la adecuación para satisfacer las necesidades de la población o la calidad de la atención, lo que depende del volumen de pacientes con enfermedad renal y del otro apoyo de la fuerza de trabajo (p. ej., disponibilidad de equipos multidisciplinarios).

Para otros proveedores de atención esenciales para el cuidado renal, existen variaciones internacionales en la distribución (disponibilidad y adecuación). En general, la escasez de proveedores fue más alta para los patólogos renales, los coordinadores de acceso vascular y los nutricionistas (el $86 \%$, el $81 \%$ y el $78 \%$ de los países reportaron escasez, respectivamente), y la escasez fue más común en los países con ingresos bajos. Pocos países (35\%) informaron una escasez de técnicos de laboratorio. Esta información destaca una importante variabilidad interregional e intrarregional en la capacidad actual de atención renal en todo el mundo. En muchos países y regiones se identificaron importantes brechas en el conocimiento, los servicios, la fuerza laboral y la capacidad para brindar una atención óptima ${ }^{4}$. Los hallazgos tienen implicaciones para el desarrollo de políticas con respecto al establecimiento de programas sólidos de atención renal, en particular para los países con ingresos bajos y medios-bajos ${ }^{46}$. El Global Kidney Health Atlas, por lo tanto, ha proporcionado un entendimiento básico de la situación de los países y las regiones con respecto a varios dominios del sistema de salud, lo que permite el monitoreo del progreso a través de la implementación de diversas estrategias dirigidas a lograr la igualdad y la calidad del cuidado del gran número de pacientes con enfermedad renal en todo el mundo.

¿Cómo podría usarse esta información para mitigar las barreras existentes para el cuidado renal? Primero, la infraestructura básica para los servicios debe fortalecerse en el nivel de atención primaria para la detección y el manejo tempranos de la LRA y la ERC en todos los países ${ }^{46}$. En segundo lugar, aunque la atención renal óptima obviamente debe enfatizar la prevención para reducir las consecuencias adversas de la enfermedad renal a nivel de la población, los países (en particular los países con ingresos bajos y con ingresos medios-bajos) deben recibir apoyo al mismo tiempo para adoptar enfoques más pragmáticos en el suministro de TRR. Por ejemplo, la diálisis peritoneal aguda podría ser una modalidad atractiva para la LRA, porque este tipo de diálisis es tan eficaz como la hemodiálisis, requiere mucha menos infraestructura y puede realizarse con soluciones y catéteres adaptados a los recursos locales ${ }^{47}$. Tercero, el trasplante de riñón debe ser fomentado a través de una mayor conciencia entre el público y los líderes políticos en todos los países, ya que esta es la modalidad clínicamente óptima de la TRR y también es costo-efectiva, siempre que los costos de la cirugía y los medicamentos a largo plazo, así como el seguimiento sean sostenibles a través de financiamiento público (y/o privado). Actualmente, la mayoría de los trasplantes de riñón se realizan en países con ingresos altos, en parte debido a la falta de recursos y conocimientos en los países con ingresos bajos y medios-bajos, así como a las prácticas culturales y la ausencia de marcos legales que rigen la donación de órganos ${ }^{48}$.

\section{Conclusión}

Las personas socialmente desfavorecidas experimentan una carga desproporcionada de enfermedad renal en todo el mundo. La provisión y el suministro de atención renal varían ampliamente en todo el mundo. Lograr la cobertura universal de salud en todo el mundo para 2030 es uno de los objetivos de desarrollo sostenible de la OMS. Aunque la cobertura de salud universal puede no incluir todos los elementos del cuidado renal en todos los países (debido a que esto generalmente es una función de factores políticos, económicos y culturales), comprender lo que es factible e importante para un país o región con un enfoque en reducir la carga y las consecuencias de la enfermedad renal serían un paso importante para lograr la equidad en la salud renal.

\section{Conflicto de intereses}

Todos los autores declararon no tener conflicto de interés 


\section{Agradecimientos}

Los autores agradecen al equipo de Global Kidney Health Atlas, M. Lunney, y M.A. Osman.

\section{Apéndice}

Los Miembros del Comité de Dirección del Día Mundial del Riñón son Philip Kam Tao Li, Guillermo Garcia-Garcia, Sharon Andreoli, Deidra Crews, Kamyar Kalantar-Zadeh, Charles Kernahan, Latha Kumaraswami, Gamal Saadi, y Luisa Strani.

\section{Bibliografía}

1. GBD 2015 DALYs and HALE Collaborators. Global, regional, and national disability-adjusted life-years (DALYs) for 315 diseases and injuries and healthy life expectancy (HALE), 1990-2015: a systematic analysis for the Global Burden of Disease Study 2015. Lancet. 2016;388:1603-58.

2. Hill NR, Fatoba ST, Oke JL, et al. Global prevalence of chronic kidney disease-a systematic review and metaanalysis. PLoS One. 2016; 11:e0158765.

3. Crews DC, Liu Y, Boulware LE. Disparities in the burden, outcomes, and care of chronic kidney disease. Curr Opin Nephrol Hypertens. 2014 23:298-305.

4. Bello AK, Levin A, Tonelli M, et al. Global Kidney Health Atlas: a report by the International Society of Nephrology on the current state of organization and structures for kidney care across the globe. https://www. theisn.org/images/ISN_advocacy/GKHAtlas_Linked_Compressed1.pdf. Published 2017. Accessed August 22, 2018.

5. Stanifer JW, Jing B, Tolan S, et al. The epidemiology of chronic kidney disease in sub-Saharan Africa: a systematic review and meta-analysis. Lancet Glob Health. 2014;2:e174-e181.

6. Davids MR, Eastwood JB, Selwood NH, Arogundade FA, Ashuntantang G, Benghanem Gharbi $M$, et al. A renal registry for Africa: first steps. Clin Kidney J. 2016;9:162-7.

7. GBD 2015 Mortality and Causes of Death Collaborators. Global, regional, and national life expectancy, all-cause mortality, and cause-specific mortality for 249 causes of death, 1980-2015: a systematic analysis for the Global Burden of Disease Study 2015. Lancet. 2016;388:1459-544.

8. Liyanage T, Ninomiya T, Jha V, et al. Worldwide access to treatment for end-stage kidney disease: a systematic review. Lancet. 2015;385: 1975-82.

9. Mehta RL, Cerda J, Burdmann EA, et al. International Society of Nephrology's 0by25 initiative for acute kidney injury (zero preventable deaths by 2025): a human rights case for nephrology. Lancet. 2015;385:2616-43.

10. Samuel SM, Palacios-Derflingher L, Tonelli M, et al. Association between First Nations ethnicity and progression to kidney failure by presence and severity of albuminuria. CMAJ. 2014;186:E86-E94.

11. Nicholas SB, Kalantar-Zadeh K, Norris KC. Racial disparities in kidney disease outcomes. Semin Nephrol. 2013;33:409-15.

12. Van den Beukel TO, de Goeij MC, Dekker FW, Siegert CE, Halbesma $\mathrm{N}$; PREPARE Study Group. Differences in progression to ESRD between black and white patients receiving predialysis care in a universal health care system. Clin J Am Soc Nephrol. 2013;8:1540-7.

13. Crews DC, Gutierrez OM, Fedewa SA, et al. Low income, community poverty and risk of end stage renal disease. BMC Nephrol. 2014;15:192.

14. Garrity $\mathrm{BH}, \mathrm{Kramer} \mathrm{H}$, Vellanki $\mathrm{K}$, et al. Time trends in the association of ESRD incidence with area-level poverty in the US population. Hemodial Int. 2016;20:78-83.

15. Parsa A, Kao WH, Xie D, et al. APOL1 risk variants, race, and progression of chronic kidney disease. N Engl J Med. 2013;369:2183-96.

16. Peralta $C A$, Bibbins-Domingo $K$, Vittinghoff $E$, et al. APOL1 genotype and race differences in incident albuminuria and renal function decline. J Am Soc Nephrol. 2016;27:887-93.

17. Correa-Rotter R. Mesoamerican nephropathy or chronic kidney disease of unknown origin. In: García- García G, Agodoa LY, Norris KC, eds. Chronic Kidney Disease in Disadvantaged Populations. Cambridge, MA: Academic Press; 2017. p. 221-8.

18. Levin A, Stevens PE, Bilous RW, et al. Kidney Disease: Improving Global Outcomes (KDIGO) CKD Work Group. KDIGO 2012 clinical practice guideline for the evaluation and management of chronic kidney disease. Kidney Int Suppl. 2013;3:1-150.
19. Chan JC, Gregg EW, Sargent J, Horton R. Reducing global diabetes burden by implementing solutions and identifying gaps: a Lancet Commission. Lancet. 2016;387:1494-5.

20. Kearney PM, Whelton M, Reynolds K, et al. Global burden of hypertension: analysis of worldwide data. Lancet. 2005;365:217-23.

21. Plantinga LC, Miller ER $3^{\text {rd }}$, Stevens LA, et al. Blood pressure control among persons without and with chronic kidney disease: US trends and risk factors 1999-2006. Hypertension. 2009;54:47-56.

22. Banerjee T, Liu Y, Crews DC. Dietary patterns and CKD progression. Blood Purif. 2016;41:117-22.

23. Johnson AE, Boulware LE, Anderson CA, et al. Perceived barriers and facilitators of using dietary modification for CKD prevention among African Americans of low socioeconomic status: a qualitative study. BMC Nephrol. 2014;15:194.

24. Crews DC, Kuczmarski MF, Miller ER $3^{\text {rd }}$, et al. Dietary habits, poverty, and chronic kidney disease in an urban population. J Ren Nutr. 2015; 25:103-10.

25. Suarez JJ, Isakova T, Anderson CA, et al. Food access, chronic kidney disease, and hypertension in the U.S. Am J Prev Med. 2015;49:912-20.

26. Crews DC, Kuczmarski MF, Grubbs V, et al. Effect of food insecurity on chronic kidney disease in lower-income Americans. Am J Nephrol. 2014;39:27-35.

27. Banerjee T, Crews DC, Wesson DE, et al. Food insecurity, CKD, and subsequent ESRD in US adults. Am J Kidney Dis. 2017;70:38-47.

28. Piccoli GB, Alrukhaimi M, Liu ZH, et al. Women and kidney disease: reflections on World Kidney Day 2018. Kidney Int. 2018;93:278-83.

29. Food and Agriculture Organization of the United Nations. The FAO hunger map 2015. http://www.fao.org/3/a-i4674e.pdf. Published 2015. Accessed August 22, 2018.

30. Shariff ZM, Khor GL. Obesity and household food insecurity: evidence from a sample of rural households in Malaysia. Eur $\mathrm{J}$ Clin Nutr. 2005;59:1049-58.

31. Popkin BM. Contemporary nutritional transition: determinants of diet and its impact on body composition. Proc Nutr Soc. 2011;70:82-91.

32. Sawhney S, Marks A, Fluck N, et al. Intermediate and long-term outcomes of survivors of acute kidney injury episodes: a large population-based cohort study. Am J Kidney Dis. 2017;69:18-28.

33. Heung M, Steffick DE, Zivin K, et al. Acute kidney injury recovery pattern and subsequent risk of CKD: an analysis of Veterans Health Administration data. Am J Kidney Dis. 2016;67:742-52.

34. Grams ME, Matsushita K, Sang Y, et al. Explaining the racial difference in AKI incidence. J Am Soc Nephrol. 2014;25:1834-41.

35. Luyckx VA, Tuttle KR, Garcia-Garcia G, et al. Reducing major risk factors for chronic kidney disease. Kidney Int Suppl. 2017;7:71-87.

36. Lewington AJ, Cerda J, Mehta RL. Raising awareness of acute kidney injury: a global perspective of a silent killer. Kidney Int. 2013;84:457-67.

37. Cervantes L, Tuot D, Raghavan R, et al. Association of emergency-only vs standard hemodialysis with mortality and health care use among undocumented immigrants with end-stage renal disease. JAMA Intern Med. 2018;178:188-95.

38. Rizvi SA, Naqvi SA, Zafar MN, Akhtar SF. A kidney transplantation model in a low-resource country: an experience from Pakistan. Kidney Int Suppl. 2013;3:236-40.

39. Roberti J, Cummings $\mathrm{A}$, Myall $\mathrm{M}$, et al. Work of being an adult patient with chronic kidney disease: a systematic review of qualitative studies. BMJ Open. 2018;8:e023507.

40. Kierans C, Padilla-Altamira C, Garcia-Garcia G, et al. When health systems are barriers to health care: challenges faced by uninsured Mexican kidney patients. PLoS One. 2013;8:e54380.

41. Murray CJ, Frenk J. A framework for assessing the performance of health systems. Bull World Health Organ. 2000;78:717-31.

42. Htay $\mathrm{H}$, Alrukhaimi M, Ashuntantang GE, et al. Global access of patients with kidney disease to health technologies and medications: findings from the Global Kidney Health Atlas project. Kidney Int Suppl. 2018;8:64-73.

43. Osman MA, Alrukhaimi M, Ashuntantang GE, et al. Global nephrology workforce: gaps and opportunities toward a sustainable kidney care system. Kidney Int Suppl. 2018;8:52-63.

44. World Health Organization. Global Health Observatory indicator views. http://apps.who.int/gho/data/node. imr\#ndx-P. Accessed August 22, 2018.

45. Harris DC, Dupuis S, Couser WG, Feehally J. Training nephrologists from developing countries: does it have a positive impact? Kidney Int Suppl. 2012;2:275-8.

46. Couser WG, Remuzzi G, Mendis S, Tonelli M. The contribution of chronic kidney disease to the global burden of major noncommunicable diseases. Kidney Int. 2011;80:1258-70.

47. Chionh CY, Soni SS, Finkelstein FO, et al. Use of peritoneal dialysis in AKI: a systematic review. Clin J Am Soc Nephrol. 2013;8:1649-60.

48. Muralidharan A, White S. The need for kidney transplantation in low- and middle-income countries in 2012: an epidemiological perspective. Transplantation. 2015;99:476-8. 